\title{
GABA Concentration Is Reduced in Visual Cortex in Schizophrenia and Correlates with Orientation-Specific Surround Suppression
}

\author{
Jong H. Yoon, ${ }^{1}$ Richard J. Maddock, ${ }^{1}$ Ariel Rokem, ${ }^{2}$ Michael A. Silver, ${ }^{2}$ Michael J. Minzenberg, ${ }^{1}$ J. Daniel Ragland, ${ }^{1}$ \\ and Cameron S. Carter ${ }^{1}$ \\ ${ }^{1}$ Department of Psychiatry and Imaging Research Center, University of California, Davis, Davis, California 95817, and ${ }^{2}$ School of Optometry and Helen Wills \\ Neuroscience Institute, University of California, Berkeley, Berkeley, California 94720
}

\begin{abstract}
The neural mechanisms underlying cognitive deficits in schizophrenia remain essentially unknown. The GABA hypothesis proposes that reduced neuronal GABA concentration and neurotransmission results in cognitive impairments in schizophrenia. However, few in vivo studies have directly examined this hypothesis. We used magnetic resonance spectroscopy (MRS) at high field to measure visual cortical GABA levels in 13 subjects with schizophrenia and 13 demographically matched healthy control subjects. We found that the schizophrenia group had an $\sim 10 \%$ reduction in GABA concentration. We further tested the GABA hypothesis by examining the relationship between visual cortical GABA levels and orientation-specific surround suppression (OSSS), a behavioral measure of visual inhibition thought to be dependent on GABAergic synaptic transmission. Previous work has shown that subjects with schizophrenia exhibit reduced OSSS of contrast discrimination (Yoon et al., 2009). For subjects with both MRS and OSSS data ( $n=16)$, we found a highly significant positive correlation $(r=0.76)$ between these variables. GABA concentration was not correlated with overall contrast discrimination performance for stimuli without a surround $(r=-0.10)$. These results suggest that a neocortical GABA deficit in subjects with schizophrenia leads to impaired cortical inhibition and that GABAergic synaptic transmission in visual cortex plays a critical role in OSSS.
\end{abstract}

\section{Introduction}

GABA has been the focus of significant interest in the search for neural mechanisms of cognitive deficits in schizophrenia. Converging evidence points to deficient GABA synthesis resulting from reduced transcription of the $67 \mathrm{kDa}$ isoform of glutamic acid decarboxylase (GAD67) within parvalbumin-staining cortical neurons (PV) (Akbarian et al., 1995; Volk et al., 2000; Hashimoto et al., 2003, 2008b). Animal studies showing decreased GABA in $\mathrm{PV}$-positive $\left(\mathrm{PV}^{+}\right)$interneurons after deletion of the GAD67 gene (Asada et al., 1997; Chattopadhyaya et al., 2007) suggest that GABA concentrations may be reduced in schizophrenia. However, there is a paucity of in vivo studies that have examined this prediction. One recent report described decreased GABA in the basal ganglia in schizophrenia but no difference in the parietooccipital or frontal lobes (Goto et al., 2009). Furthermore, the GABA hypothesis predicts that abnormalities in GABAergic neurons should result in impairments in dependent cognitive processes in schizophrenia (Cho et al., 2006).

In the first part of our study, we measured in vivo GABA concentrations in the visual cortex of subjects with schizophrenia

\footnotetext{
Received Dec. 12, 2009; revised Jan. 28, 2010; accepted Feb. 3, 2010.

This study has been funded by the National Alliance for Research on Schizophrenia and Depression Foundation and National Institutes of Health/National Institute of Mental Health.

C.S.C. has been a paid consultant for Merck, Eli Lilly and Co., and Roche. R.J.M. has been a paid consultant for Pfizer.

Correspondence should be addressed to Jong H. Yoon, Imaging Research Center, University of California, Davis, 4701 X Street, Sacramento, CA 95817. E-mail: jhyyoon@ucdavis.edu.

DOI:10.1523/JNEUROSCI.6158-09.2010

Copyright $\odot 2010$ the authors $\quad 0270-6474 / 10 / 303777-05 \$ 15.00 / 0$
}

and healthy control subjects using high-field magnetic resonance spectroscopy (MRS). Recent histological evidence suggests that GABA deficits in schizophrenia are pan-cortical and not restricted to a particular region (Hashimoto et al., 2008a). Specifically, reduced GAD67 mRNA has been reported in a number of neocortical regions, including visual cortex. Consequently, we hypothesized that GABA concentration in schizophrenia would be reduced in visual cortex.

Measurement of GABA concentrations allowed us to assess the functional significance of a GABA deficit for behavior. We examined the relationship between GABA concentrations and performance on orientation-specific surround suppression (OSSS), a process thought to rely on GABAergic neurotransmission in the visual cortex. Surround suppression is most pronounced for center and surround stimuli sharing the same stimulus orientation (Blakemore and Tobin, 1972; Xing and Heeger, 2001). Animal and human studies (Blakemore and Tobin, 1972; Cavanaugh et al., 2002b; Haynes et al., 2003; Zenger-Landolt and Heeger, 2003) have demonstrated neural correlates of surround suppression in primary visual cortex, an area known to contain many orientation-selective neurons. Current models of OSSS postulate a critical role for GABAergic inhibitory neurons in early visual cortex (Angelucci and Bressloff, 2006; Schwabe et al., 2006). However, there is currently little direct evidence supporting this role for GABA. We have reported previously that subjects with schizophrenia exhibit diminished OSSS compared with healthy control subjects (Yoon et al., 2009). In the present study, we correlated GABA levels obtained in the 
Table 1. Subject demographics and patient clinical characteristics

\begin{tabular}{|c|c|c|c|c|c|}
\hline & \multicolumn{2}{|c|}{ Patient $(n=13)$} & \multicolumn{2}{|c|}{ Control $(n=13)$} & \multirow[b]{2}{*}{$p$ value } \\
\hline & Mean & SD & Mean & SD & \\
\hline Age (years) & 27.5 & 8.8 & 28.1 & 8.2 & 0.87 \\
\hline Gender (\% male) & 85 & & 85 & & \\
\hline Education (years) & 14.0 & 1.4 & 15.9 & 1.7 & 0.01 \\
\hline Parental education (years) & 14.5 & 2.4 & 15.0 & 3.4 & 0.69 \\
\hline On medications (\%) & 62 & & & & \\
\hline CPZ equivalents (mg) & 433 & 228 & & & \\
\hline GAF & 49.8 & 12.7 & & & \\
\hline BPRS & 41.4 & 10.5 & & & \\
\hline SAPS & 5.8 & 4.4 & & & \\
\hline SANS & 10.2 & 3.3 & & & \\
\hline
\end{tabular}

GAF, Global Assessment of Functioning; BPRS, Brief Psychiatric Rating Scale; SAPS, Scale for the Assessment of Positive Symptoms; SANS, Scale for the Assessment of Negative Symptoms.

first part of the study with subjects' performance on the OSSS task. We hypothesized that there would be a positive correlation between visual cortical GABA levels and magnitude of OSSS.

\section{Materials and Methods}

Subjects. We studied 13 subjects with schizophrenia and 13 healthy control subjects matched on basic demographic factors (Table 1). All patients were clinically stable and recruited as outpatients. They consisted of a mixture of chronic and recent onset schizophrenia. Diagnosis was made by master or doctoral-level clinicians using Structured Clinical Interview for DSM-IV and confirmed by consensus conference. Exclusion criteria were as follows: intelligence quotient $<70$, drug or alcohol dependence or abuse within 3 months of testing, a positive urine drug screen on the day of testing, medical or neurological illness affecting brain function, or history of significant head trauma. Exclusion criteria for controls were lifetime diagnosis of Axis I disorder or first-degree relative with a psychotic disorder. Subscores from the Brief Psychiatric Rating Scale, Scale for the Assessment of Negative Symptoms, and Scale for the Assessment of Positive Symptoms were used to derive indices for the following three major domains of symptoms: reality distortion, disorganization, and negative symptoms (Barch et al., 2003). At the time of testing, five patients had not been taking antipsychotics for at least 1 month. Four of these subjects had minimal previous exposure to any antipsychotic ( $<2$ weeks of treatment). Among the medicated patients, one subject was on a typical antipsychotic and seven were on atypical antipsychotics. No control subject was taking any psychotropic medication. This study was approved by the Institutional Review Board at the University of California, Davis, and all subjects provided written informed consent for all procedures.

Spectroscopy methods. GABA was measured with proton MRS using a Siemens Trio 3T magnetic resonance imaging (MRI) system. Paired surface coils (Nova Medical) were positioned under the occiput. A $35 \times$ $30 \times 25 \mathrm{~mm}$ voxel was centered on the calcarine sulci bilaterally, with its posterior border $7 \mathrm{~mm}$ anterior to the dura. A single-volume MEGA point-resolved spectroscopy (PRESS) J-difference spectral editing sequence measured total GABA (Mescher et al., 1998). Alternating subspectra were obtained with and without a frequency-selective inversion pulse applied to the GABA C3 resonance. The subspectra were subtracted to generate a difference spectrum containing an upright total GABA signal at $\sim 3.0 \mathrm{ppm}$. The following scanning parameters were used: repetition time, $1500 \mathrm{~ms}$; echo time, $68 \mathrm{~ms}$; edit frequency $1.9 \mathrm{ppm}$; delta frequency, -1.7; edit pulse bandwidth, $45 \mathrm{~Hz}$; number of excitations, 256; scan duration, $6.5 \mathrm{~min}$. Four consecutive scans were acquired for each subject from the same volume location.

Using jMRUI software (Stefan et al., 2009), all spectra were phase aligned with reference to water, zero filled from 1024 to 4096, apodized with a $4 \mathrm{~Hz}$ Gaussian filter, and frequency aligned to creatine $(\mathrm{Cr})$ at 3.02 ppm. Peak integration was used to quantify total GABA $(2.99 \pm 0.12$ $\mathrm{ppm})$ and combined glutamine plus glutamate (Glx) (3.76 $\pm 0.11 \mathrm{ppm})$ in the difference spectra and creatine $(3.02 \pm 0.09 \mathrm{ppm})$ in the summed spectra. The ratio of total GABA/total creatine signal was used for hypothesis testing. Normalizing to creatine reduces intersubject variance attributable to differences in global signal strength and CSF fraction within the voxel and has been shown to yield reliable GABA concentration estimates (Bogner et al., 2009). Significant subject motion during acquisition causes residual creatine signal to obscure the GABA signal in the difference spectra. One of us (R.J.M.) examined each scan for such artifacts while blinded to group membership. Of a total of 52 scans for each group, 13 patient and 8 control scans were excluded.

Visual psychophysics. For the present study, we used psychophysical results from 16 subjects (seven controls and nine patients) who also participated in the MRS experiment. Data from 13 subjects are from a previously reported study (Yoon et al., 2009). The task was a contrast discrimination task (Fig. 1) in which one segment within the annulus contained lower contrast than the other segments under three conditions: no surround (NS), vertically oriented surround [parallel surround (PS)], and horizontally oriented surround [orthogonal surround (OS)]. The stimulus was a circular patch consisting of a contrast-reversing (4 $\mathrm{Hz}$ ), grayscale sinusoidal grating with a spatial frequency of 1.1 cycles/ ${ }^{\circ}$. The stimulus was divided into annulus and surround regions (when present) by concentric black lines. The inner and outer radii of the annulus were 2.9 and $5.6^{\circ}$, respectively. The surround region contained the remainder of the stimulus: the central portion, extending from the central fixation point to the inner border of the annulus, and the outer portion, extending from the outer border of the annulus to an eccentricity of $9.2^{\circ}$. In half the trials, a segment with lower contrast was present, and the location of this target within the annulus varied randomly. In the remaining trials, no target was present, i.e., all segments displayed the same contrast. Task difficulty was adjusted using an adaptive staircase. The ratio of contrast discrimination thresholds of the PS and OS conditions (PS/OS) provides the most direct measure of OSSS, because the these stimuli are identical except for the orientation of the surround relative to the annulus. Additionally, the PS/OS ratio is the measure most commonly used in the single-unit electrophysiology literature to characterize correlates of OSSS. For these reasons, the PS/OS ratio was used to correlate OSSS with GABA concentrations. All statistical comparisons were conducted using two-tailed tests of significance.

\section{Results}

\section{MRS GABA measurements}

Clearly resolved MRS peaks for both total GABA and Glx were observed in all participants. Group-averaged spectra for GABA and Glx and their means, normalized by the Cr peak integration value for each subject, are displayed in Figure $2 \mathrm{~A}$. A significant difference between patients and controls in GABA/Cr variance was not present (Levene's test for equality of variance, $p=0.66$ ). Patients displayed significantly smaller GABA/Cr ratios compared with healthy control subjects (controls, 0.105; schizophrenia group, 0.094; $p<0.05)$. Glx/Cr peaks were equivalent across groups (controls, 0.102 ; schizophrenia group, $0.103 ; p=0.88$ ) (Fig. 2B). To examine the possible confound of antipsychotic medications, we conducted two analyses. First, the correlation between daily chlorpromazine equivalents (CPZ) and GABA/Cr was $r=0.003$. Second, we performed an ANCOVA of GABA/Cr with $\mathrm{CPZ}$ as a covariate, with all subjects, including controls, not taking neuroleptics being assigned a value of $0 \mathrm{CPZ}$. This analysis revealed nonsignificant covariance with medication dosage $\left(F_{(1,23)}<0.001, p=0.988\right)$ and a trend toward main effect of group $\left(F_{(1,23)}=3.48, p=0.075\right)$.

\section{Correlation of visual cortical GABA levels and OSSS}

For the 16 subjects with both MRS and surround suppression data, we correlated visual cortical GABA/Cr values with behavioral measures of surround suppression. Previous work has shown that surround suppression is selective for stimulus orientation, with greater suppression in the PS relative to the OS condition (Yoon et al., 2009). The reduction in surround suppression in schizophrenia is also orientation specific: it is present 

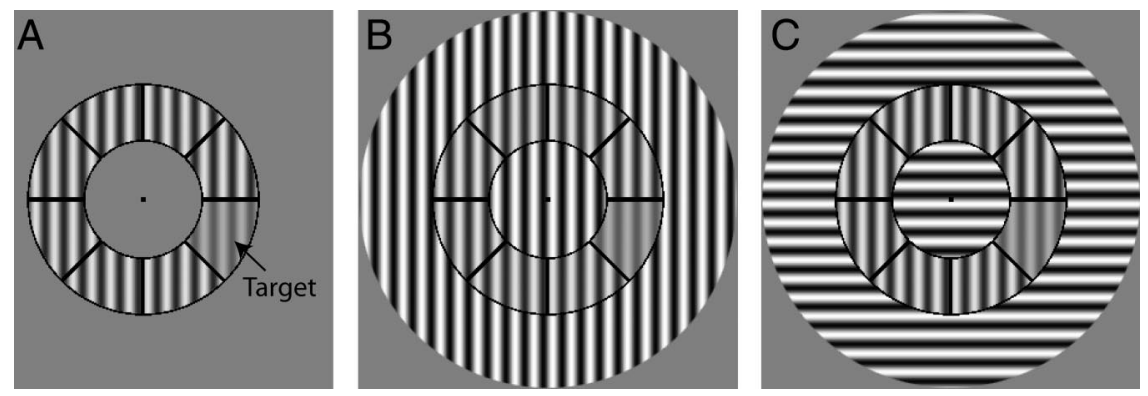

Figure 1. Surround suppression stimuli. Subjects indicated whether they perceived a difference in contrast between any one segment (target) and the other segments in the annulus under three conditions: $\boldsymbol{A}, \mathrm{NS} ; \boldsymbol{B}, \mathrm{PS} ;$ and $\boldsymbol{C}, 0 \mathrm{OS}$. The annulus is identical in all three conditions in this figure.

A

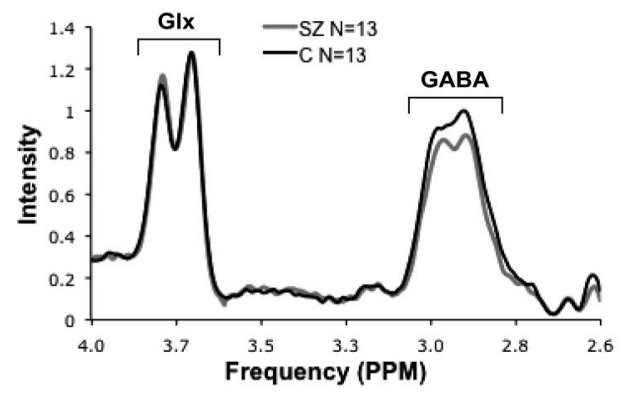

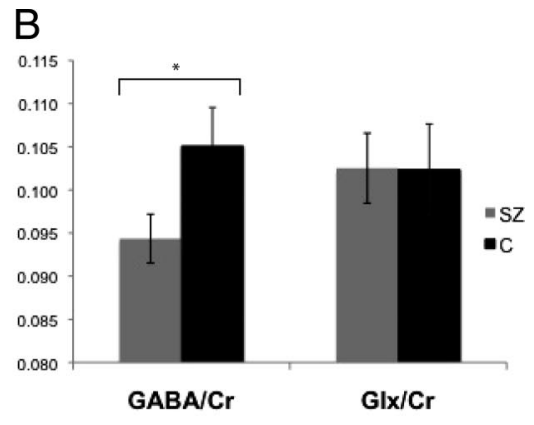

Figure 2. Nuclear magnetic resonance spectra results. $A$, Group-averaged, creatine-normalized Glx and total GABA spectra. SZ, Schizophrenia; $C$, controls. $\boldsymbol{B}$, Integrals of the creatine-normalized GABA and Glx peaks. ${ }^{*} p<0.05$. Error bars denote SDs.

A

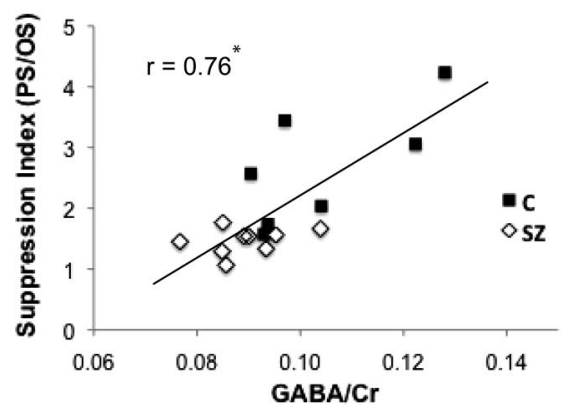

B

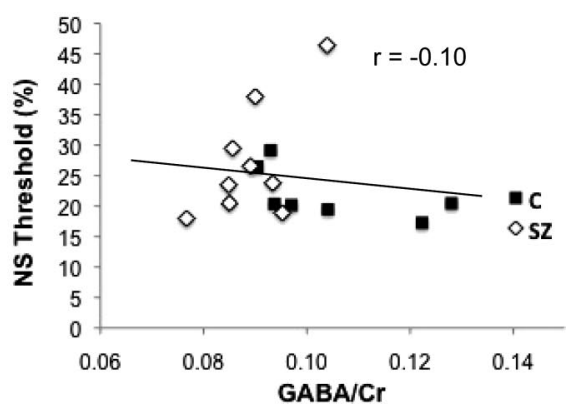

Figure 3. Visual cortical GABA levels correlate with OSSS. GABA/Cr plotted against the contrast discrimination threshold in the PS condition normalized by the OS threshold $\left({ }^{*} p=0.001\right)(\boldsymbol{A})$ and threshold in the NS condition $(\boldsymbol{B})$. SZ, Schizophrenia; $\boldsymbol{C}$, controls.

for the PS but not the OS condition (Yoon et al., 2009), indicating a deficit in neural circuits that are selective for stimulus orientation. In the present study, there was also a significant group difference in OSSS, as indexed by PS/OS $(p=0.003)$. We related $\mathrm{GABA} / \mathrm{Cr}$ levels to PS/OS because this index is the most direct measure of the component of surround suppression that is orientation specific (Fig. 3A). This correlation was highly significant $(r=0.76, p=0.001)$. In contrast, the correlation of GABA concentration and NS performance was not significant $(r=-0.10$, $p=0.70$ ), indicating that the correlation with OSSS was not attributable to contrast discrimination performance.

To test whether the significant correlations were driven by outliers, we performed a jackknife analysis. The data were resampled $n$ times ( $n$ indicates number of subjects), and, in each resampling, one subject was excluded and the correlation was then computed. The range of GABA-PS/OS correlations was $0.58-0.83$ (for all samples, $p<0.05$ ). In contrast, the range of
GABA-NS threshold correlations was $-0.32-0.04$ (for all samples, $p>0.1$ ).

To evaluate the possibility that the GABA-PS/OS correlation was the result of group differences in these variables, we conducted the following analyses. ANCOVA of the PS/OS ratio with GABA/ $\mathrm{Cr}$ as a covariate and group as a factor assessed the effect of GABA concentration on surround suppression while taking into account group differences in GABA levels. The effect of the GABA covariate was significant $\left(F_{(1,13)}=8.50, p=0.01\right)$, and there was a trend toward a main effect of group $\left(F_{(1,13)}=4.28, p=0.059\right)$. The power of the GABA covariate was 0.77 . We accounted for group differences in both GABA and PS/OS ratio by subtracting the group means from the subject's values and then testing the association between the normalized data. This correlation was significant $(r=0.62, p<0.05)$. Using $r=0.62$ as the estimate of the effect size, power was 0.77 . Finally, we conducted GABA-PS/OS correlations separately for each group (controls, $r=0.71$, $p=0.077$; schizophrenia, $r=0.32, p=$ $0.40)$. A power analysis showed that, in control subjects, we would need $n=12$ to have power of 0.80 at a significance level of $p=0.05$.

There were no significant correlations between GABA concentration and any clinical measure.

\section{Discussion}

This is one of the first published studies examining in vivo brain GABA levels in schizophrenia. We found significantly reduced visual cortical GABA in the schizophrenia group. We also found a significant positive correlation between GABA levels and magnitude of OSSS. Together, these findings support the GABA deficit hypothesis of cognitive impairments in schizophrenia: that reduced neocortical GABA neurotransmission in schizophrenia leads to impairments in cognitive processes involving inhibition. These results also suggest that relatively simple behavioral tasks such as surround suppression may serve as behavioral assays of inhibitory dysfunction in schizophrenia.

Although our conclusions are derived from measurements of GABA in visual cortex and a task thought to involve visual cortical GABAergic transmission, it is likely that analogous abnormalities generalize to other cortical areas. Histological studies have documented similar deficits in GABAergic markers in a number of cortical regions in schizophrenia (Hashimoto et al., 2008a). In addition, there is substantial evidence that the morphological subtypes of cortical neurons and their basic laminar and tangential connectivity are conserved across cortical areas (Douglas and Martin, 2004).

Our group and others have previously demonstrated impaired visual inhibition in schizophrenia (Must et al., 2004; Dakin et al., 
2005; Tadin et al., 2006; Yoon et al., 2009). Although these previous studies are consistent with the GABA deficit hypothesis, they did not measure GABA concentrations. The present study, by demonstrating both a deficit in GABA concentrations in schizophrenia and a strong association between GABA levels and a behavioral measure of visual inhibition, provides a direct link between GABA dysfunction and cognitive deficits in schizophrenia.

Our finding of reduced GABA is consistent with the animal and postmortem literature. The GAD67 synthesizes the overwhelming majority of neural GABA (Asada et al., 1997; Chattopadhyaya et al., 2007). In schizophrenia, postmortem studies have documented an $\sim 50 \%$ reduction in the number of $\mathrm{PV}^{+}$neurons with detectable levels of GAD67 mRNA in the dorsolateral prefrontal cortex, in which $\mathrm{PV}^{+}$neurons constitute $\sim 25 \%$ of all GABAergic neurons (Hashimoto et al., 2003). These results suggest that GAD67 deficits in schizophrenia should translate into measurable reductions in GABA concentration.

A number of studies have addressed issues of sensitivity and validity of MRS measurements of brain GABA. A recent study demonstrated high test-retest reliability of the MEGA-PRESS method, particularly when using the creatine normalization procedure (Bogner et al., 2009). Other studies have demonstrated that the MEGA-PRESS method has sufficient sensitivity to detect changes in GABA levels resulting from interventions known to alter GABA concentrations (Verhoeff et al., 1999; Floyer-Lea et al., 2006).

We did not find a group difference in Glx values. Previous studies measuring glutamate or Glx in prefrontal regions have yielded inconsistent results (Theberge et al., 2002; van Elst et al., 2005; Ohrmann et al., 2007; Galińska et al., 2009; Lutkenhoff et al., 2010). We are not aware of any MRS studies of glutamate or Glx in visual cortex in schizophrenia. Our Glx results control for generalized measurement error because the potential sources of bias toward finding group differences for Glx are nearly identical to those for GABA, e.g., sensitivity to head movement and differences in the proportions of gray matter within the volume. Equivalent Glx measurements suggest that the group differences in GABA are specific.

Dopamine blocking agents have been shown to lower extracellular levels of GABA (Bourdelais and Deutch, 1994). Although the specific mechanisms are likely complex, with $\mathrm{D}_{2}$ and $\mathrm{D}_{1}$ receptor activation promoting decreased and increased GABA release, respectively (Seamans et al., 2001), antipsychotics could be contributing to the lower GABA MRS measurements in subjects with schizophrenia. Several lines of evidence, however, suggest that this may not be the case. Monkey and humans studies have shown similar patterns of GAD67 deficits in subjects with and without exposure to antipsychotics (Volk et al., 2000; Hashimoto et al., 2008b). In our sample, the trend toward the persistence of the group difference in GABA concentration after covarying for medication dosage and the low correlation between antipsychotic dosage and GABA concentration argue against a significant effect. Nonetheless, future studies testing a larger sample of medication-naive subjects is required to fully address this medication confound.

The fact that surround suppression exhibits selectivity for stimulus orientation for both behavior (Xing and Heeger, 2001; Yoon et al., 2009) and primary visual cortex V1 neuron responses (Blakemore and Tobin, 1972; Cavanaugh et al., 2002a) suggests an early visual cortical locus. In addition, functional MRI studies (Zenger-Landolt and Heeger, 2003) have demonstrated correlates of surround suppression in primary visual cortex. However, the spatial (Angelucci et al., 2002; Cavanaugh et al., 2002a) and temporal (Bair et al., 2003) properties of surround suppression suggest a critical role for feedback projections from extrastriate cortex (Angelucci and Bressloff, 2006). Because these feedback projections are excitatory, current models propose that they modulate surround suppression via inhibitory GABAergic interneurons (Cavanaugh et al., 2002a; Schwabe et al., 2006; Smith, 2006). These models receive support from intracellular recordings of V1 neurons, in which presentation of a surround stimulus elicited a transient increase in inhibitory conductance, followed by a sustained reduction in both excitatory and inhibitory conductance, all of which were orientation selective (Ozeki et al., 2009).

A previous study assessed the effects of local iontophoresis of the $\mathrm{GABA}_{\mathrm{A}}$ receptor antagonist bicuculline on surround suppression (Ozeki et al., 2004). Bicuculline reduced the magnitude of surround suppression of individual V1 neuronal responses $\sim 12 \%$. However, it is difficult to interpret the effects of local changes in inhibition, because surround suppression likely involves circuits outside the region of drug administration. In contrast, we measured global GABA concentrations in visual cortex and demonstrated its strong correlation with the magnitude of OSSS.

An important issue not addressed by this study is the primacy of GABA deficits in schizophrenia, particularly in reference to glutamatergic dysfunction. Some have proposed that GABA abnormalities are downstream effects of impaired NMDA receptor-mediated neurotransmission, which results in reduced glutamatergic drive on activity-dependent GABA synthesis (Lewis and Gonzalez-Burgos, 2006).

In summary, we found reduced GABA concentrations in the visual cortex of subjects with schizophrenia. GABA concentrations were highly correlated with a behavioral measure of visual inhibition, such that higher GABA concentration predicted greater OSSS. These findings provide the first in vivo evidence of a deficit in cortical GABA in schizophrenia and support the GABA deficit hypothesis of cognitive deficits in this disorder. Additional study of the GABA system is likely to provide important insights into the neural mechanisms of these cognitive deficits.

\section{References}

Akbarian S, Kim JJ, Potkin SG, Hagman JO, Tafazzoli A, Bunney WE Jr, Jones EG (1995) Gene expression for glutamic acid decarboxylase is reduced without loss of neurons in prefrontal cortex of schizophrenics. Arch Gen Psychiatry 52:258-266.

Angelucci A, Bressloff PC (2006) Contribution of feedforward, lateral and feedback connections to the classical receptive field center and extraclassical receptive field surround of primate V1 neurons. Prog Brain Res 154:93-120.

Angelucci A, Levitt JB, Walton EJ, Hupe JM, Bullier J, Lund JS (2002) Circuits for local and global signal integration in primary visual cortex. J Neurosci 22:8633-8646.

Asada H, Kawamura Y, Maruyama K, Kume H, Ding RG, Kanbara N, Kuzume H, Sanbo M, Yagi T, Obata K (1997) Cleft palate and decreased brain gamma-aminobutyric acid in mice lacking the $67-\mathrm{kDa}$ isoform of glutamic acid decarboxylase. Proc Natl Acad Sci U S A 94:6496-6499.

Bair W, Cavanaugh JR, Movshon JA (2003) Time course and time-distance relationships for surround suppression in macaque V1 neurons. J Neurosci 23:7690-7701.

Barch DM, Carter CS, MacDonald AW 3rd, Braver TS, Cohen JD (2003) Context-processing deficits in schizophrenia: diagnostic specificity, 4-week course, and relationships to clinical symptoms. J Abnorm Psychol 112:132-143.

Blakemore C, Tobin EA (1972) Lateral inhibition between orientation detectors in the cat's visual cortex. Exp Brain Res 15:439-440. 
Bogner W, Gruber S, Doelken M, Stadlbauer A, Ganslandt O, Boettcher U, Trattnig S, Doerfler A, Stefan H, Hammen T (2009) In vivo quantification of intracerebral GABA by single-voxel (1)H-MRS: how reproducible are the results? Eur J Radiol. Advance online publication. Retrieved Feb. 26, 2010. doi:10.1016/j.ejrad.2009.01.014.

Bourdelais AJ, Deutch AY (1994) The effects of haloperidol and clozapine on extracellular GABA levels in the prefrontal cortex of the rat: an in vivo microdialysis study. Cereb Cortex 4:69-77.

Cavanaugh JR, Bair W, Movshon JA (2002a) Nature and interaction of signals from the receptive field center and surround in macaque V1 neurons. J Neurophysiol 88:2530-2546.

Cavanaugh JR, Bair W, Movshon JA (2002b) Selectivity and spatial distribution of signals from the receptive field surround in macaque V1 neurons. J Neurophysiol 88:2547-2556.

Chattopadhyaya B, Di Cristo G, Wu CZ, Knott G, Kuhlman S, Fu Y, Palmiter RD, Huang ZJ (2007) GAD67-mediated GABA synthesis and signaling regulate inhibitory synaptic innervation in the visual cortex. Neuron 54:889-903.

Cho RY, Konecky RO, Carter CS (2006) Impairments in frontal cortical gamma synchrony and cognitive control in schizophrenia. Proc Natl Acad Sci U S A 103:19878-19883.

Dakin S, Carlin P, Hemsley D (2005) Weak suppression of visual context in chronic schizophrenia. Curr Biol 15:R822-R824.

Douglas RJ, Martin KA (2004) Neuronal circuits of the neocortex. Annu Rev Neurosci 27:419-451.

Floyer-Lea A, Wylezinska M, Kincses T, Matthews PM (2006) Rapid modulation of GABA concentration in human sensorimotor cortex during motor learning. J Neurophysiol 95:1639-1644.

Galińska B, Szulc A, Tarasów E, Kubas B, Dzienis W, Czernikiewicz A, Walecki J (2009) Duration of untreated psychosis and proton magnetic resonance spectroscopy (1H-MRS) findings in first-episode schizophrenia. Med Sci Monit 15:CR82-CR88.

Goto N, Yoshimura R, Moriya J, Kakeda S, Ueda N, Ikenouchi-Sugita A, Umene-Nakano W, Hayashi K, Oonari N, Korogi Y, Nakamura J (2009) Reduction of brain gamma-aminobutyric acid (GABA) concentrations in early-stage schizophrenia patients: 3T proton MRS study. Schizophr Res 112:192-193.

Hashimoto T, Volk DW, Eggan SM, Mirnics K, Pierri JN, Sun Z, Sampson AR, Lewis DA (2003) Gene expression deficits in a subclass of GABA neurons in the prefrontal cortex of subjects with schizophrenia. J Neurosci 23:6315-6326.

Hashimoto T, Bazmi HH, Mirnics K, Wu Q, Sampson AR, Lewis DA (2008a) Conserved regional patterns of GABA-related transcript expression in the neocortex of subjects with schizophrenia. Am J Psychiatry 165:479-489.

Hashimoto T, Arion D, Unger T, Maldonado-Avilés JG, Morris HM, Volk DW, Mirnics K, Lewis DA (2008b) Alterations in GABA-related transcriptome in the dorsolateral prefrontal cortex of subjects with schizophrenia. Mol Psychiatry 13:147-161.

Haynes JD, Roth G, Stadler M, Heinze HJ (2003) Neuromagnetic correlates of perceived contrast in primary visual cortex. J Neurophysiol 89:2655-2666.

Lewis DA, Gonzalez-Burgos G (2006) Pathophysiologically based treatment interventions in schizophrenia. Nat Med 12:1016-1022.

Lutkenhoff ES, van Erp TG, Thomas MA, Therman S, Manninen M, Huttunen MO, Kaprio J, Lönnqvist J, O’Neill J, Cannon TD (2010) Proton MRS in twin pairs discordant for schizophrenia. Mol Psychiatry 15:308-318.

Mescher M, Merkle H, Kirsch J, Garwood M, Gruetter R (1998) Simulta- neous in vivo spectral editing and water suppression. NMR Biomed 11:266-272.

Must A, Janka Z, Benedek G, Kéri S (2004) Reduced facilitation effect of collinear flankers on contrast detection reveals impaired lateral connectivity in the visual cortex of schizophrenia patients. Neurosci Lett 357:131-134.

Ohrmann P, Siegmund A, Suslow T, Pedersen A, Spitzberg K, Kersting A, Rothermundt M, Arolt V, Heindel W, Pfleiderer B (2007) Cognitive impairment and in vivo metabolites in first-episode neuroleptic-naive and chronic medicated schizophrenic patients: a proton magnetic resonance spectroscopy study. J Psychiatr Res 41:625-634.

Ozeki H, Sadakane O, Akasaki T, Naito T, Shimegi S, Sato H (2004) Relationship between excitation and inhibition underlying size tuning and contextual response modulation in the cat primary visual cortex. J Neurosci 24:1428-1438.

Ozeki H, Finn IM, Schaffer ES, Miller KD, Ferster D (2009) Inhibitory stabilization of the cortical network underlies visual surround suppression. Neuron 62:578-592.

Schwabe L, Obermayer K, Angelucci A, Bressloff PC (2006) The role of feedback in shaping the extra-classical receptive field of cortical neurons: a recurrent network model. J Neurosci 26:9117-9129.

Seamans JK, Durstewitz D, Christie BR, Stevens CF, Sejnowski TJ (2001) Dopamine D1/D5 receptor modulation of excitatory synaptic inputs to layer V prefrontal cortex neurons. Proc Natl Acad Sci U S A 98:301-306.

Smith MA (2006) Surround suppression in the early visual system. J Neurosci 26:3624-3625.

Stefan D, Di Cesare F, Andrasescu A, Popa E, Lazariev A, Vescovo E, Strback O, Williams S, Starcuk Z, Cabanas M, van Ormondt D, Graveron-Demilly D (2009) Quantitation of magnetic resonance spectroscopy signals: the mJRUI software package. Meas Sci Technol 20:104035-104044.

Tadin D, Kim J, Doop ML, Gibson C, Lappin JS, Blake R, Park S (2006) Weakened center-surround interactions in visual motion processing in schizophrenia. J Neurosci 26:11403-11412.

Théberge J, Bartha R, Drost DJ, Menon RS, Malla A, Takhar J, Neufeld RW Rogers J, Pavlosky W, Schaefer B, Densmore M, Al-Semaan Y, Williamson PC (2002) Glutamate and glutamine measured with 4.0 T proton MRS in nevertreated patients with schizophrenia and healthy volunteers. Am J Psychiatry 159:1944-1946

van Elst LT, Valerius G, Büchert M, Thiel T, Rüsch N, Bubl E, Hennig J, Ebert D, Olbrich HM (2005) Increased prefrontal and hippocampal glutamate concentration in schizophrenia: evidence from a magnetic resonance spectroscopy study. Biol Psychiatry 58:724-730.

Verhoeff NP, Petroff OA, Hyder F, Zoghbi SS, Fujita M, Rajeevan N, Rothman DL, Seibyl JP, Mattson RH, Innis RB (1999) Effects of vigabatrin on the GABAergic system as determined by [123I]iomazenil SPECT and GABA MRS. Epilepsia 40:1433-1438.

Volk DW, Austin MC, Pierri JN, Sampson AR, Lewis DA (2000) Decreased glutamic acid decarboxylase67 messenger RNA expression in a subset of prefrontal cortical gamma-aminobutyric acid neurons in subjects with schizophrenia. Arch Gen Psychiatry 57:237-245.

Xing J, Heeger DJ (2001) Measurement and modeling of center-surround suppression and enhancement. Vision Res 41:571-583.

Yoon JH, Rokem AS, Silver MA, Minzenberg MJ, Ursu S, Ragland JD, Carter CS (2009) Diminished orientation-specific surround suppression of visual processing in schizophrenia. Schizophr Bull 35:1078-1084.

Zenger-Landolt B, Heeger DJ (2003) Response suppression in v1 agrees with psychophysics of surround masking. J Neurosci 23:6884-6893. 